\title{
LA DINAMICA DEI DOVERI E LE FORME DELLA GIUSTIZIA NEL MODELLO POLITICO DEL GIOVANE GROZIO
}

\author{
di Ilaria Pizza
}

Questo lavoro si propone di muovere dalla disamina delle diverse forme di diritto individuate da Ugo Grozio nel suo De jure praedae (1604$08)^{1}$, al fine di poter, poi, mettere a fuoco le tipologie di giustizia e la dinamica dei doveri, che operano all'interno della Respublica qui descritta.

Tuttavia, una lettura critica di questo primo trattato di stampo internazionalistico del giusnaturalista olandese non può prescindere dal tenere debitamente in conto che negli anni in cui l'autore si dedica alla stesura di esso, è ancora legato ad una concezione volontaristica del diritto di chiara impronta calvinista, destinata ad essere poi superata in seguito alla sua successiva adesione all' arminianesimo ${ }^{2}$. Nel commen-

Università degli Studi di Napoli 'Federico II'.

1 Va qui ricordato che il De jure praedae commentarius non è mai stato pubblicato nella sua interezza durante la vita di Grozio. Com'è noto, unicamente il capitolo XII dell'opera vide la luce con il nome di Mare liberum, prima in forma anonima nel 1608, poi con il nome dell'autore nel 1612. Sebbene la corrispondenza groziana consenta di far risalire il testo originale del trattato al 1604-1605, studi recenti confermano che esso sia stato sottoposto dall'autore ad una sostanziale revisione dal gennaio del 1607 fino ai mesi di novembre o dicembre del 1608, presumibilmente in previsione della successiva pubblicazione del Mare liberum. In proposito, si veda M.J. VAn ITtersum, Preparing Mare Liberum for the Press: Hugo Grotius' Rewriting of Chapter 12 of De Iure Praedae in November-December 1608, in "Grotiana", n. 26-28, 2005-2007, pp. 246-280. L'unica copia esistente del trattato - conosciuta come Manoscritto BPL 917 - fu acquistata ad un'asta dall'Università di Leida nel 1864, in seguito alla morte dell'ultimo erede maschio del giurista olandese, e venne data alle stampe quattro anni dopo da un umanista della stessa Università, Hendrik Gerard Hamaker. Sul punto si rimanda a M.J. VAN ITTERSuM, The wise man is never merely a private citizen: The Roman Stoa in Hugo Grotius 'De Jure praedae (1604-1608), in "History of European Ideas", n. 36, 2010, p. 4.

2 Come è noto, nel pensiero maturo del giusnaturalista olandese, che trova piena formulazione nel De jure belli ac pacis, "l'ordine naturale è considerato espressione di strutture 
tario sullo jus praedae, infatti, il giovane avvocato olandese non ha ancora realizzato il processo di autonomizzazione morale del diritto dalla teologia, che troverà, invece, compimento nel capolavoro della maturità, il De jure belli ac pacis (1625). Pertanto, la struttura espositiva del trattato sul diritto di preda è tutta incentrata sull'affermazione del carattere prescrittivo della volontà di Dio, manifestatasi nel creato.

Ed è appunto a partire dal primato del volere divino che viene qui tratteggiata quella peculiare classificazione delle forme di diritto, che funge da presupposto della visione antropologica e politica giovanile del pensatore di Delft, per analizzare la quale va innanzitutto rilevata la totale assenza nel De jure praedae della distinzione tra diritto naturale e diritto volontario, vale a dire tra un diritto che abbia la propria fonte unicamente nella natura razionale dell'uomo ed uno derivante, viceversa, dai comandi di una libera volontà, umana o divina ${ }^{3}$. Questa distinzione costituirà, invece, la premessa di carattere giuridico dell'intera costruzione teorico-politica del De jure belli ac pacis ${ }^{4}$. Più precisamente, il motivo di tale assenza risiede nel fatto che nel trattato giovanile di Grozio il sommo volere di Dio rappresenta il fondamento

essenzialistiche, distinte da una fondazione di carattere teologico, cui è insita una ratio unica ed universale": V. FIORILlo, Tra egoismo e socialità. Il giusnaturalismo di Samuel Pufendorf, Napoli, Jovene, 1992, p. 84. L'influenza di tale ontologia razionale sulla visione antropologica e, di conseguenza, sulla ricostruzione della nascita dello Stato nell'opus magnum groziano è stata ben messa in luce da G. STIENING nel suo recente saggio dal titolo Natur und Staat. Politische Anthropologie bei Marsilius von Padua und Hugo Grotius - mit einem Seitenblich auf die Antike, in S. LePsius, F. VollhardT, O. BACH (Hrsg.), "Von der Allegorie zur Empirie. Natur im Rechtsdenken des Spätmittelalters und der Frühen Neuzeit", Berlin, Erich Schmidt Verlag, 2018, soprattutto pp. 212-224. La notevole distanza, che separa la concezione ontologica e giuridica del De jure praedae da quella del De jure belli ac pacis, è chiaramente evidenziata da L. WinkeL, Problems of legal systematization from De Jure praedae to De Jure belli ac pacis. De Jure praedae Chapter II and the Prolegomena of De Jure belli ac pacis compared, in "Grotiana", n. 26-28, 2005-2007, pp. 61-78.

3 Cfr. H. Grotil, De jure belli ac pacis. Libri tres, In quibus Jus Naturae \& Gentium, item Juris Publici praecipua explicantur, Amsterdam, Janssonio-Waesbergios, 1720, I, I, $\mathrm{X}, 1$, p. 10: “Jus naturale est dictatum rectae rationis, indicans actui alicui, ex ejus convenientia aut disconvenentia cum ipsa natura rationali ac sociali, inesse moralem turpitudinem, aut necessitatem moralem, ac consequenter ab auctore naturae Deo talem actum aut vetari, aut praecipi”; e H. GrotII, De jure belli ac pacis, cit., I, I, XIII, p. 17: “Alteram juris speciem esse diximus jus voluntarium, quod ex voluntate originem ducit: estque vel humanum vel divinum".

4 Cfr. H. Grotil, De jure belli ac pacis, cit., I, I, IX, 2, p. 9: "Juris ita accepti optima partitio est, quae apud Aristotelem exstat, ut sit aliud jus naturale, aliud voluntarium". 
originario del diritto, come attesta la prima regola che l'essere umano riconosce, secondo cui "quod Deus se velle significarit, id jus est"s. Da questo principio essenziale, che indica come diritto quello che il Signore manifesta di volere, derivano poi otto ulteriori Regulae, specificanti gli altri tipi di fonti del diritto ${ }^{6}$, vale a dire il consensus gentium, il volere del singolo e quello della Respublica, del magistrato e di tutte le Respublicae. Tredici Leges precisano, infine, il contenuto di queste regole, dando vita ad un apparato giuridico organizzato, per così dire, secondo una serie di cerchi concentrici unificati dalla volontà divina, in maniera tale che "the larger the circles, the more general the problems they solve, so that the closer circles might concentrate on more particular questions without touching again the problems of the foundation"7. Pertanto, l'autore può descrivere lo sviluppo lineare dello jus naturae come effetto diretto dell'intervento ordinatore di Dio8.

In particolare, Grozio distingue uno jus naturae primarium, comune a tutti gli ordini della natura, da uno jus naturae secundarium, detto anche jus gentium primarium ${ }^{9}$, peculiare dell'uomo, quale unica creatura dotata di ragione ${ }^{10}$. Egli ritiene, infatti, che Dio palesi la sua volontà sia nell'or-

5 Cfr. H. Grotil, De jure praedae commentarius, a cura di H.G. HAMAKER, Hagae Comitum, Martinum Nijhoff, 1868, cap. I, pp. 7-8 (corsivo nell'originale).

6 Cfr. H. GrotII, De jure praedae commentarius, cit., cap. II, pp. 7-30. Per ulteriori osservazioni sul sistema di Regulae e Leges presente nel capitolo II del De jure praedae si rimanda, tra i tanti, solo a M. ScatTola, Law, War and Method in the Commentary on The Law of Prize by Hugo Grotius, in "Grotiana", n. 26-28, 2005-2007, pp. 95-103 e a F. TodESCAN, 'Sequuntur Dogmatica De Iure Praedae'. Law and Theology in Grotius's use of Sources in De Iure Praedae, in "Grotiana", n. 26-28, 2005-2007, pp. 281-309. Benjamin Straumann evidenzia, inoltre, come, da un punto di vista metodologico, il trattato giovanile di Grozio costituisca un tentativo di introdurre una nuova dottrina delle fonti del diritto servendosi del metodo proprio dei retori classici, e soprattutto di Cicerone. In proposito, si veda B. STRAUMANN, 'Ancient Caesarian Lawers'in a State of Nature: Roman Tradition and Natural Rights in Hugo Grotius's 'De iure praedae', in "Political Theory", n. 3, 2006, pp. 328-350.

7 Cfr. M. Scattola, Law, War and Method in the Commentary on The Law of Prize by Hugo Grotius, cit., p. 96.

8 Cfr. P. Negro, Note a U. Grozio, I fondamenti del diritto. Antologia, Napoli, Editoriale Scientifica, 1997, p. 257, n. 9.

9 Cfr. H. Grotil, De jure praedae commentarius, cit., cap. II, p. 12.

10 Va ricordato che, per il giovane autore, oltre ad un diritto delle genti primario (o diritto naturale secondario), esiste anche un diritto delle genti secondario. Come si è detto, la prima forma di diritto - più specificamente lo jus naturae secundarium o jus gentium primarium - coincide con quelle norme iscritte da Dio nell'umanità e comprensibili esclu- 
dine interno alla creazione ${ }^{11}$, sia mediante la ratio umana, capace di comprendere il funzionamento del mondo. Altrimenti detto, dalla volontà divina, fonte primaria del diritto, trae origine lo jus naturae primarium, ossia la legge comune a tutte le creature; a sua volta, da tale diritto deriva, "through the mediation of the collective will of men, taken as rational creatures, the jus naturale secundarium" 12 .

sivamente mediante la ratio, per cui quando tutti i popoli riconoscono necessariamente come giusti determinati comportamenti è perché essi sono conformi alla loro specifica natura. Il giusnaturalista olandese è, però, consapevole che non tutte le norme stabilite dal diritto delle genti hanno un carattere di necessità morale, sicché egli distingue dallo jus gentium primarium lo jus gentium secundarium, che è detto essere, invece, "quoddam jus mixtum ex jure gentium et civili": H. GroTII, De jure praedae commentarius, cit., cap. II, p. 26. Il diritto delle genti secondario, dunque, indica l'insieme delle norme positive, tratte sia dal diritto delle genti primario, sia dal diritto civile dei singoli Stati, posto dalla volontà dei cittadini, al rispetto delle quali si sono volontariamente obbligate le Respublicae in vista del bene comune. In altri termini, esso è costituito dalle convenzioni e dagli accordi, che vigono in ambito internazionale, come ad esempio l'inviolabilità dei legati ed il dovere di seppellire i morti. In proposito, si veda H. GROTII, De jure praedae commentarius, cit., cap. II, pp. 22-23. Va sottolineato che, sebbene lo jus gentium secundarium possa contenere delle leggi che sono riprese dallo jus civile degli Stati, queste due forme di diritto - precisamente lo jus civile e lo jus gentium secundarium - sono ben diverse tra loro. Difatti, il diritto delle genti secondario contiene in parte dei comandi, che, provenendo dallo jus naturae primarium, hanno la pretesa di regolamentare l'umanità nel suo complesso, ovvero tutti gli esseri dotati di ragione. Al contrario, Grozio sin dal primo capitolo dell'opera rimarca il carattere contingente delle leges scriptae (jus civile) delle diverse Respublicae, le quali, mutando nel tempo e nello spazio, non possono, ad esempio, essere utili ad emettere giudizi sulle contese tra popoli diversi. Sul punto si rimanda ad H. GrotII, De jure praedae commentarius, cit., cap. I, p. 5.

${ }^{11}$ La distinzione tra le due diverse tipologie di diritto naturale, che Grozio riprende dalla tradizione scolastica, presuppone la convinzione, successivamente criticata dallo stesso autore nel De jure belli ac pacis, che una qualche forma di diritto, e specificamente il diritto naturale primario, possa vigere anche per gli esseri irrazionali. In proposito, cfr. H. GrotII, De jure belli ac pacis, cit., I, Prol. 17, pp. XII-XIII. Ciò perché, secondo il Grozio del De jure praedae, la volontà legislatrice divina si manifesterebbe non tanto negli oracoli o nei segni straordinari, quanto soprattutto (maxime) nella creantis intentio, vale a dire nell'ordo naturalis sorretto dallo jus naturae. Su ciò si veda H. GrotiI, De jure praedae commentarius, cit., cap. II, p. 8. Si può così comprendere che "Grotius's voluntarism is of a particular kind, for it is consistent with the nature of a thing and with the nature of the world as a whole": M. SCATtOlA, Law, War and Method in the Commentary on The Law of Prize by Hugo Grotius, cit., p. 96. In questa visione della natura traspare l'influenza sul pensiero del giovane giurista da parte della dottrina panteistica stoica, la quale - com'è risaputo - intende la divinità come $\lambda o ́$ yos, insito nella materia per darle forma, quale sua causa efficiente.

${ }^{12}$ Cfr. F. Todescan, 'Sequuntur Dogmatica De Iure Praedae'. Law and Theology in Grotius's use of Sources in "De Iure Praedae”, cit., p. 299. 
In tale prospettiva, il diritto naturale primario si esprime attraverso l'istinto di autoconservazione, che accomuna tutti gli esseri della terra, così come anche le cose inanimate ${ }^{13}$, manifestandosi mediante quelle proprietà naturali insite nel creato, al fine di condurlo al suo bene ${ }^{14}$. Tali proprietà naturali si traducono negli animali e, quindi, anche negli uomini in due particolari affectiones, la fuga e l'appetitus, che spingono rispettivamente a scappare dai mali ed a ricercare quanto possa giovare $^{15}$. Tanto è vero che il contenuto dello jus naturae primarium si specifica nelle prime due leggi di natura, le quali obbligano tutte le creature all'amor sui, attribuendo loro il diritto alla salvaguardia di sé e a procacciarsi quanto sia utile alla sopravvivenza: "Prior. Vitam tueri et declinare nocitura liceat. Altera: Adjungere sibi quae ad vivendum sunt utilia eaque retinere liceat" 16 .

A partire da tali presupposti, Grozio può abbozzare già in questo scritto giovanile la sua dottrina del suum, secondo cui gli esseri umani sarebbero in possesso sin dalla nascita di alcuni bona di ordine prioritario (prior et dignitor ordo), ossia la vita e l'integrità fisica ${ }^{17}$, la viola-

${ }^{13}$ Cfr. H. GrotII, De jure belli ac pacis, cit., cap. II, p. 9, ove Grozio sottolinea come il fatto che tutta la natura voglia se stessa salva e beata sia evidente non solo negli uomini e nelle bestie, ma persino "in rebus inanimis".

${ }^{14} \mathrm{Cfr}$. H. GROTII, De jure belli ac pacis, cit., cap. II, p. 9: "Cum igitur res conditas Deus esse voluerit, proprietates quasdam naturales singulis indidit, quibus ipsum illud esse conserveretur et quibus ad bonum suum unumquodque, velut ex prima originis lege, duceretur".

${ }^{15}$ Cfr. H. GRotII, De jure praedae commentarius, cit., cap. II, p. 10: "Sunt autem haec duplicia, alia enim boni, alia mali ad nos rationem habent: quod et affectiones indicant duae, quas non homini soli sed animantibus cunctis natura indidit, fuga et appetitus".

${ }^{16}$ Cfr. H. GrotII, De jure praedae commentarius, cit., cap. II, p. 10 (corsivo nell'originale). La formulazione della prima legge di natura è seguita dal rimando ad alcuni passi tratti da svariate opere ciceroniane, in cui, nell'ottica del tardo stoicismo, si ribatte la naturalità dell'istinto di autoconservazione. A questo riguardo, si veda CiCERONE, Dei doveri, a cura di D. ARFELLI, Bologna, Zanichelli, 1991, I, IV, 1, p. 12; ID., De finibus bonorum et malorum, in ID., "Opere politiche e filosofiche", trad. it. a cura di N. MARINONE, Torino, Utet, 2005, vol. II, IV, 7, 16, p. 311 e V, 9, 24, p. 387; infine, ID., Orazione in difesa di Milone, commentata da V. Menghini, Torino, Giovanni Chiantore Editore, 1923, IV, 10, pp. 9-10. La profonda influenza, che la dottrina del diritto naturale d'impronta ciceroniana ha avuto sul De jure praedae, è ben messa in evidenza da B. Straumann, 'Ancient Caesarian Lawers' in a State of Nature: Roman Tradition and Natural Rights in Hugo Grotius's 'De iure praedae', cit., pp. 328-350.

${ }^{17}$ Cfr. H. GrotII, De jure praedae commentarius, cit., cap. II, p. 11: "Haec autem bona malaque in summa duorum sunt generum. Prioris et dignitorisque ordinis sunt ea 
zione dei quali legittimerebbe sempre una reazione violenta contro l'offensore. Tuttavia, per il giusnaturalista, una medesima tutela si estenderebbe anche a quei beni definiti di ordine secondario, perché collocati all'esterno del corpo dell'uomo, ma necessari al suo benessere ed alla sua conservazione ${ }^{18}$. La sfera originaria del suum - caratterizzata, come si è detto, dall'inviolabilità - risulta, così, essere potenzialmente ampliabile ad includere ciò che sia utile alla sopravvivenza, come conferma il dettato della Lex II con il suo "adjungere sibi"19. Del resto, l'autore precisa che le cose più vili sono state concesse da Dio in uso a quelle più insigni, come le erbe e le piante alle bestie, e quest'ultime, unitamente a tutti i beni dell'universo, all'uomo, in quanto creatura che eccelle in dignità sulle altre ${ }^{20}$. Ed è proprio in riferimento alla descrizione biblica della creazione, ripresa da Grozio, che la capacità appropriativa dell'uomo si chiarisce ancor di più. Difatti, egli ritiene che Dio abbia donato all'umanità il possesso in comune dei beni della terra, sicché ognuno avrebbe diritto a godere di quello che gli necessita, conformemente all'amor sui, che gli è instillato sin dalla nascita.

Tale amore di sé, assicurato dal dettato delle prime due leggi di natura, rientranti nello jus naturae primarium - e, dunque, comune a tutti gli esseri della terra - non è, però, ritenuto sufficiente per la salvaguardia del creato dal Signore che, perciò, volle che ognuno, oltre che al proprio benessere, si interessasse anche a quello altrui ${ }^{21}$. Perciò, Iddio infuse nelle sue creature l'amor alterius ${ }^{22}$, il quale diviene particolarmente evidente nell'essere umano grazie al dono esclusivo della ragione ${ }^{23}$.

quae corpus ipsum attingunt, ut in malorum censu morset huic affinis membrorum mutilatio et morbi; in bonorum vita cum integritate corporis et bona valetudine".

${ }^{18} \mathrm{Cfr}$. H. GrotII, De jure praedae commentarius, cit., cap. II, p. 10: "Secundae classis quae sunt, ea ad res pertinent extra nos positas, nobis tamen utiles aut inutiles, tristes aut jucundas".

${ }^{19} \mathrm{Si}$ veda ancora H. GrotII, De jure praedae commentarius, cit., cap. II, p. 10.

${ }^{20}$ Cfr. H. GrotII, De jure praedae commentarius, cit., cap. II, p. 11: "Omnino autem viliora praestantiorum usibus concessa, ut plantae herbaeque bestiis, bestiae et res in universum omnes homini, ut qui dignitate rebus omnibus conditis praecellat".

${ }^{21}$ Cfr. H. GrotII, De jure praedae commentarius, cit., cap. II, p. 11: “At vero non satis conservationi operum suorum provisum Deus credidit, si suam duntaxat incolumitatem cuique commendaret, nisi et rem alteram alterius commodis vellet consulere, ut cuncta inter se velut aeterno foedere consentirent".

22 Cfr. H. Grotil, De jure praedae commentarius, cit., cap. II, p. 11: “Duplex (...) est amor: sui et alterius, quorum ille cupidinis, hic amicitiae dicitur".

${ }^{23}$ Cfr. H. GrotII, De jure praedae commentarius, cit., cap. II, p. 11. 
Proprio questo amore verso l'altro costituisce l'oggetto principale della seconda tipologia di diritto naturale indicata da Grozio, ossia dello jus naturae secundarium, il quale, a differenza del diritto naturale primario, operante secondo delle "proprietates qu[aedam] naturales" 24 insite in tutte le creature, presuppone l'intervento dell'intelligenza. Tale diritto naturale secondario deve, infatti, essere compreso, e non solo intuito dall'uomo, a cui mostra come agire conformemente alla sua specifica natura, contraddistinta appunto dalla razionalità25. In particolare, le prime due leggi naturali sul bene altrui - la terza e la quarta rientranti nello jus naturae secundarium, prescrivono rispettivamente di astenersi da quanto appartenga all'altro (Lex III), comandando che "ne quis alterum laedat"26, e di non occupare ciò che sia già occupato dall'altro (Lex IV), poiché "ne quis occupet alteri occupata"27. È evidente, quindi, che la $I I I$ e la $I V L e x$ circoscrivono il giusto limite dell'amor sui, comandato dalle prime due leggi naturali 28 .

Inoltre, dalla considerazione secondo cui tutti gli uomini sarebbero dotati di capacità razionale, consegue che si debba supporre essere giusta una norma su cui vi sia un comune consenso, in quanto ciò vorrà dire che essa è stata razionalmente valutata da tutti come conforme alla natura umana, ovvero adeguata al volere divino insito nell' ordo mundis e tradotto nelle leggi naturali. Ecco perché Grozio specifica che i pre-

${ }^{24}$ Cfr. H. GRotil, De jure praedae commentarius, cit., cap. II, p. 9.

${ }^{25} \mathrm{Nel}$ chiarire il nesso tra jus naturae e jus gentium nel pensiero del giovane Grozio, Franco Todescan osserva che quando questi scrive del diritto delle genti, non fa riferimento a nessun teologo, bensì a giuristi e filosofi, come Cicerone. E citando l'Arpinate, il giusnaturalista olandese parrebbe ritenere che tale diritto sia dedotto per mezzo della ragione, per cui "it (...) seems founded more on ratio than on voluntas": F. TODESCAN, 'Sequuntur Dogmatica De Iure Praedae'. Law and Theology in Grotius's use of Sources in De Iure Praedae, cit., p. 301. Del resto, per Grozio, lo jus gentium è, sì, parte del diritto naturale si pensi allo jus gentium primarium - ma è anche messo in relazione al consenso, il quale è a sua volta collegato sia alla ragione che alla volontà. Difatti, "consent is not possible only because it is wanted, but it is also agreement on what is recognised as good, true, and rational': F. Todescan, 'Sequuntur Dogmatica De Iure Praedae'. Law and Theology in Grotius's use of Sources in De Iure Praedae, cit., p. 301.

${ }^{26} \mathrm{Cfr}$. H. GROTII, De jure praedae commentarius, cit., cap. II, p. 13 (corsivo nell'originale).

${ }^{27}$ Cfr. H. GrotII, De jure praedae commentarius, cit., cap. II, p. 14 (corsivo nell'originale).

${ }^{28}$ Cfr. H. GRotII, De jure praedae commentarius, cit., cap. II, p. 13: "Ex regula igitur prima et secunda leges duae procedunt de bono alieno, quae prioribus de bono suo respondent, easque justo limite circumscribunt". 
cetti dello jus naturae secundarium hanno valore di diritto se acclamati dal consensus gentium ${ }^{29}$, come chiarisce la Regola II, per la quale "quod consensus hominum velle cunctos significaverit, id jus est" proprio il consensus gentium attesta la naturale socialità umana. Difatti, è con il diritto naturale secondario che incomincia, per il giusnaturalista olandese, quella "mediam justitiam, quae humano generi propria est" 31 . In altri termini, la giustizia propria dell'uomo consisterebbe nel mantenersi nel mezzo tra l'interesse personale e quello altrui ${ }^{32}$.

In particolare, seguendo l'insegnamento aristotelico ${ }^{33}$, Grozio sostiene qui che la giustizia, in quanto virtù sociale volta a regolare le relazioni interpersonali, possa presentare due forme. La giustizia proporzionale o assegnatrice (assignatrix) - definita distributiva dallo Stagirita - si avvale appunto di una proporzione geometrica per assegnare $\mathrm{i}$ beni a più soggetti in base al merito ${ }^{34}$. Essa, quindi, opera riferendo le parti al tutto. La giustizia compensativa (compensatrix), invece - corrispondente alla giustizia commutativa aristotelica - guarda non a ciò che è comune, bensì a quello che è proprio di ognuno, così da poter equiparare le parti tra loro. Pertanto, essa ha il compito di con-

${ }^{29}$ Cfr. M. VILLEY, La formazione del pensiero giuridico moderno, Milano, Jaca Book, 1986, p. 518.

${ }^{30}$ Cfr. H. GRotII, De jure praedae commentarius, cit., cap. II, p. 12 (corsivo nell'originale).

${ }^{31}$ Cfr. H. Grotil, De jure praedae commentarius, cit., cap. II, p. 13.

32 Il questo suo primo trattato giusnaturalistico Grozio guarda esplicitamente al libro V dell'Etica Nicomachea per evidenziare come la giustizia sia, a suo avviso, una via di mezzo tra ciò che dobbiamo agli altri e ciò che dobbiamo a noi stessi. In particolare, egli rinvia a quel passo dell'opera, in cui Aristotele sostiene che "l'agire giustamente è intermedio tra compiere ingiustizia e subirla, infatti la prima cosa corrisponde ad avere troppo, la seconda ad avere troppo poco": ARISTOTELE, Etica Nicomachea, a cura di C. Natali, Roma-Bari, Laterza, 2009, V, 9, 30, p. 195. Come è noto, nel De jure belli ac pacis, invece, Grozio ricusa la concezione aristotelica dello justum come medietà, a favore di una nozione prettamente negativa della giustizia, consistente nell'astensione dal suum altrui. In proposito, si veda, ad esempio, H. GrotII, De jure belli ac pacis, cit., I, Prol. 44, pp. XXVIIIXXIX: "injustitia non aliam naturam habeat, quam alieni usurpationem".

${ }^{33} \mathrm{Al}$ contrario, nel De jure belli ac pacis Grozio, negando che la distinzione tra giustizia distributiva e commutativa potesse essere ricondotta unicamente ad una differente proporzione - geometrica per la prima ed aritmetica per la seconda - sostiene, piuttosto, che la justitia vada distinta in expletrix ed attributrix in base alla diversa materia cui essa si riferisce, poiché nel primo caso essa ha ad oggetto ciò che è necessariamente proprio di ognuno (suum), nel secondo, invece, ciò che va convenientemente riconosciuto ai soggetti in base al loro merito. Sul punto cfr. H. GROTII, De jure belli ac pacis, cit., I, I, VIII, 1, p. 7.

${ }^{34} \mathrm{Cfr}$. H. Grotil, De jure praedae commentarius, cit., cap. II, pp. 14-15. 
servare i beni di ciascuno e di riparare i mali ${ }^{35}$, conformemente al dettato delle due successive leggi di natura, le quali prescrivono che " $m a$ lefacta corrigenda"36 (Lex V) e "benefacta repensanda" 37 (Lex VI). Più specificamente, le Leges $V$ e $V I$ intervengono a ristabilire la giusta proporzione tra gli uomini ogni qualvolta un amore di sé falso e disordinato ${ }^{38}$ li induca ad attribuirsi più del dovuto, compromettendo così con dissidi e tumulti l'ordine sociale 39 .

Ed è appunto il problema del limite delle proprie pretese nei confronti dell'altro, ovvero dei propri diritti, che, costituendo il punto di partenza dell'analisi groziana della natura socievole dell'uomo, conduce il giovane giusnaturalista ad affrontare il tema della nascita della Respublica. Non a caso l'autore costruisce l'intero sistema dei rapporti intersoggettivi tanto nella condizione di natura quanto nello Stato prendendo le mosse dalla sua dottrina dell'appropriazione. Quest'ultima si radica sulla tesi, per cui siccome ogni uomo riceve in dono da Dio in comune con gli altri tutto il creato, egli avrà diritto a far suo ciò di cui abbisogna. La naturale titolarità ad avere diritti, propria degli esseri umani, è attestata dal dettato della seconda lex naturae, che - come si è osservato - consente di procurarsi e trattenere ciò che è utile a vivere, accordando al singolo la facoltà di ampliare la sfera di liceità individuale mediante l'inclusione di beni esterni, a cui viene estesa la stessa protezione ideale riservata alla propria persona. Grozio ritiene, tuttavia, che di tali beni si potrà godere solo rispettando l'obbligo naturale di non ledere la sfera del suum altrui, così come comandato dalla terza e dalla quarta legge di natura, giacché, in caso contrario, il comportamento dell'offensore dovrà essere corretto con la forza (Lex V). Pertanto, essendo la realtà ordinata in maniera tale che al diritto primario alla sopravvivenza si affianchi il diritto alle cose necessarie alla so-

${ }^{35}$ Cfr. H. Grotil, De jure praedae commentarius, cit., cap. II, p. 15.

${ }^{36}$ Cfr. H. GrotII, De jure praedae commentarius, cit., cap. II, p. 15 (corsivo nell'originale).

${ }^{37}$ Cfr. H. GrotII, De jure praedae commentarius, cit., cap. II, p. 15 (corsivo nell'originale).

${ }^{38}$ Cfr. H. GRotII, De jure praedae commentarius, cit., cap. II, p. 23.

39 Cfr. H. GrotII, De jure praedae commentarius, cit., cap. II, p. 23: "Nam cum homines abrepti saepe non vero sed falso atque inordinato sui amore, qui mali omnis causa est, id quod revera erat plus habere crederent aequalitatem esse et hinc dissidia ac tumultus orirentur". 
pravvivenza stessa, ne consegue che la perfezione di questo ordine richieda "che Dio instilli nelle sue creature il rispetto per l'altro, che consiste anche nel rispetto della proprietà altrui" 40 .

A questo proposito, il giusnaturalista olandese istituisce un interessante analogia tra i concetti di proprietà e di libertà, sostenendo che "quod Libertas in actionibus idem est Dominium in rebus" 41 . In tal modo, egli ravvisa nel diritto di utilizzare a proprio piacimento un bene un'estensione della libertà naturale dell'uomo di disporre del proprio corpo, giacché entrambi, sia il corpo che il bene, rientrano nella sfera del suum ${ }^{42}$. L'essere umano, quindi, avverte ciò che gli appartiene come proprio al pari delle sue membra e, pertanto, si sente egualmente indotto a proteggerlo. Il Signore, difatti, ha creato l'uomo "liberum suique juris"43, appunto affinché egli potesse agire ed utilizzare le sue cose senza soggiacere all'arbitrio altrui, così come conferma il consensus gentium ${ }^{44}$.

In conseguenza di ciò, la Regula III prevede che "quod se quisque velle significaverit, id in eum jus est" 45 . È così stabilito che vada con-

40 Cfr. P. Negro, Note a U. Grozio, I fondamenti del diritto. Antologia, cit., p. 260, n. 17.

${ }^{41}$ Cfr. H. GrotII, De jure praedae commentarius, cit, cap. II, p. 172 ("Dominium" in corsivo nell'originale).

${ }^{42}$ Sulla dottrina del suum, posta da Grozio a fondamento della sua nozione di proprietà, si rinvia, nell'ambito della letteratura sul tema: a R. BRANDT, Eigentumstheorien von Grotius bis Kant, Stuttgart-Bad Cannstatt, Freidrich Frommann Verlag, 1974, su Grozio, cap. I, pp. 31-49; K. OliveCrONA, Il "suum" secondo la dottrina del diritto naturale; K. Olivecrona, I due livelli della teoria del diritto naturale, entrambi contenuti in K. OLIVECRONA, "La realtà del diritto. Antologia di scritti", a cura di S. CASTIGLIONE, C. FARALLI, M. RIPOLI, Torino, Giappichelli Editore, 2000, rispettivamente a pp. 355-362 e a pp. 363388 , nonché del medesimo autore cfr. anche La struttura dell'ordinamento giuridico, trad. it. di E. Pattaro, Milano, Etas Kompass, in particolare pp. 292-310. Su questo tema mi sia consentito, inoltre, di rinviare a I. PIzzA, Alcune osservazioni sul fondamento della proprietà nel giusnaturalismo di Ugo Grozio, in P. Bellini, F. SCiACCA, E.S. Storace (a cura), "Miti, simboli e potere. Scritti in onore di Claudio Bonvecchio", Milano, Alboversorio, 2018, pp. 357-366. Sulla concezione proprietaria, caratteristica dell'età moderna, sono, infine, di imprescindibile riferimento i volumi di P. GROsSI, La proprietà e le proprietà nell'officina dello storico, Napoli, Editoriale Scientifica, 2006, L'inaugurazione della proprietà moderna, Napoli, Guida Editori, 1980, ed, infine, Il dominio e le cose. Percezioni medievali e moderne dei diritti reali, Milano, Giuffrè, 1992.

${ }^{43} \mathrm{Cfr}$. H. Groti, De jure praedae commentarius, cit., cap. II, p. 18 (corsivo nell'originale).

${ }^{44}$ Cfr. H. Grotil, De jure praedae commentarius, cit., cap. II, p. 18: "Fecit enim Deus

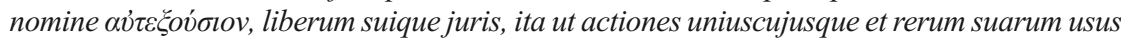
ipsius, non alieno arbitrio subjacerent, idemque gentium omnium consensu approbatur".

${ }^{45}$ Cfr. H. GrotII, De jure praedae commentarius, cit., cap. II, p. 18 (corsivo nell'originale). 
siderato diritto per il singolo ciò che egli manifesta di volere. Tale regola costituisce il presupposto del contrattualismo groziano, giacché proprio mediante la manifestazione delle volontà convergenti di più soggetti avviene la stipulazione del patto, con cui nasce lo Stato ${ }^{46}$.

Va rilevato, tuttavia, che nella visione antropologica groziana, al contrario che nel pensiero di Thomas Hobbes ${ }^{47}$, l'origine contrattuale della comunità politica si accompagna alla convinzione di una naturale socialità degli esseri umani. La società umana - frutto di un sentimento naturale di affetto per i propri simili comune a tutto il creato, e rafforzata nell'uomo dal dettato dello jus naturae secundarium - precede, infatti, il costituirsi del corpo politico. Quest'ultimo è introdotto - come si vedrà - allo scopo di assicurare la concordia e la tranquillità pubblica ${ }^{48}$, messe in pericolo nello stato di natura principalmente dalla malizia di molti uomini, derivante, a sua volta, dalla loro mente corrotta ${ }^{49}$. Tant'è che all'interno del sistema di diritto naturale illustrato da Grozio, le Leges $I I I$ e $I V$, che - come si è sopra menzionato - obbligano al ri-

${ }^{46}$ Cfr. H. GrotII, De jure praedae commentarius, cit., cap. II, p. 19.

${ }^{47}$ Com'è risaputo, nella sua analisi antropologica Hobbes individua due postulati sicurissimi della natura umana, ovvero il desiderio naturale (cupiditas naturalis), per cui ciascuno richiede per sé l'uso di cose che sono in comune, e la ragione naturale (ratio naturalis), che induce ad evitare una morte violenta, la quale rappresenta il più grande dei mali naturali. Sul punto si veda T. HobBES, De Cive. Elementi filosofici sul cittadino, a cura di T. MAGRI, Roma, Editori Riuniti, 2005, Lettera dedicatoria, p. 6. Con il primo postulato Hobbes nega la tradizione culturale prevalente - ancora condivisa da Grozio - che, facendo capo all'insegnamento aristotelico, esaltava la socievolezza naturale dell'uomo. Difatti, il filosofo inglese ritiene che l'essere umano non sia un animale politico incline, per natura, alla socialità - come voleva, invece, lo Stagirita - bensì sia naturalmente portato all'egoismo e all'avversione verso gli altri. Il movente che spinge l'uomo a stabilire rapporti sociali con i suoi simili è, quindi, unicamente la paura di perdere il più importante dei beni, ossia la vita, sicché si deve credere che "l'origine delle grandi e durevoli società [debba] essere stata non la mutua simpatia degli uomini, ma il reciproco timore": T. HoBBES, De Cive. Elementi filosofici sul cittadino, cit., I, 3, p. 23. D'altronde, "one of the most salient differences between Grotius and Hobbes consists in the picture they respectively drew of the conditions in the state of nature and the status which self-interest enjoys therein. While according to Hobbes there is nothing morally wrong with purely self-interested behavior, Grotius argues that acting solely in the pursuit of self-interest is not only morally but also legally wrong in the state of nature": B. STRAUMAnN, 'Ancient Caesarian Lawers' in a State of Nature: Roman Tradition and Natural Rights in Hugo Grotius's 'De iure praedae', cit., pp. 337-338.

${ }^{48}$ Cfr. H. GrotII, De jure praedae commentarius, cit, cap. II, p. 23.

${ }^{49}$ Cfr. H. GrotII, De jure praedae commentarius, cit, cap. II, p. 23. 
spetto della persona e dei beni altrui, costituiscono il presupposto normativo, su cui riposano le successive leggi naturali disciplinanti l'esistenza comune dei cittadini all'interno dello Stato, nonché il rapporto tra governanti e governati.

Come sinora si è visto, infatti, già nella condizione prepolitica, secondo Grozio, si manifesta la giustizia quale virtù sociale indicante il giusto mezzo tra quanto è dovuto a sé stesso e quanto agli altri. L'autore, pertanto, individua specificamente negli obblighi nei confronti del prossimo, stabiliti dal diritto naturale secondario (Leges III e IV), il giusto limite (justus limes) agli officia adversus se ipsum, sanciti, invece, dalle prime due leggi di natura. È evidente, quindi, che, mentre si avverte istintivamente ciò che è bene per sé, grazie a due affectiones naturali (fuga e appetitus), occorre, invece, l'intervento della comprensione razionale per contenere l'utile personale, al fine di rispettare il proprio simile. La consapevolezza intellettiva della necessità della cura del benessere altrui diviene così il carattere discriminante della natura umana. Solo l'uomo può cogliere, infatti, il contenuto delle successive quattro leggi naturali, che - come si è visto - comandano di non nuocere all'altro (legge III), di non occupare ciò che è occupato da altri (legge IV), di correggere i crimini (legge V) ${ }^{50}$ e di ricambiare i benefici (legge VI). Eppure, queste leggi si ripromettono il bene delle diverse persone, non quello della collettività derivante dalla loro unione ${ }^{51}$.

${ }^{50}$ L'importanza attribuita al prossimo diviene evidente soprattutto nel discorso sulla pena, introdotto dalla Lex $V$, che, comandando la correzione dei crimini, indica la liceità secondo natura del sottoporre a sanzione chi leda un diritto altrui. In proposito, è interessante osservare come per Grozio il fine della pena vada ricercato sempre non nell'interesse del singolo soggetto leso, bensì in quello dell'intera comunità, poiché "pertinent autem ad omnes quodammodo injuria etiam uni illata, ob exemplum maxime, sicut corporis interest membra habere sana, praecipue ob contagium": H. GROTII, De jure praedae commentarius, cit., cap. II, p. 16. Ciò comporta che nella Respublica due sono i generi di pena di pertinenza della totalità dei cittadini, ovvero il castigo (castigatio), che tenta di correggere e rendere più utile alla collettività colui che debba essere punito, e l'esempio (exemplum), con il quale, invece, attraverso il timore di una simile sanzione, si inducono gli altri cittadini ad astenersi dal peccare. Oltre ad una finalità correttiva e ad una dissuasiva, la pena, però, ha anche un ulteriore obiettivo, che è indicato specificamente nella sicurezza di tutti, "quia necesse est, si omnes bene faciant, neminem male pati": H. GROTII, De jure praedae commentarius, cit., cap. II, pp. 16-17.

${ }^{51}$ Cfr. H. GrotII, De jure praedae commentarius, cit., cap. II, p. 21: "Spectant itaque hae leges [Lex VII e Lex VIII] bonum quidem commune, non tamen ut diversorum, sicut leges tertii ordinis, sed ut unius atque adeo ut suum". 
Con l'ingresso nella società civile, invece, l'utilità personale e la cura per gli altri si fondono, affinché l'interesse per la sicurezza comune prevalga sulle spinte egoistiche di ciascuno. È appunto in nome del benessere collettivo che, secondo Grozio, sorgono le prime Respublicae, all'interno delle quali la vita è disciplinata da ulteriori leggi naturali, prima ancora che dal diritto positivo ${ }^{52}$. Sicché ai precetti naturali precedentemente indicati se ne aggiungono di nuovi, vòlti specificamente a disciplinare il comportamento umano all'interno della comunità politica, l'origine della quale è brevemente descritta da Grozio.

In particolare, con la sua teoria della nascita dello Stato, l'autore vuole principalmente illustrare come all'interno di un sistema di diritto naturale, in cui il presupposto di ogni relazione sociale è dato dall'aequitas naturale, ossia dall'eguaglianza nel godimento da parte di ciascuno del proprio suum, si giunga ad aderire ad un'unione sociale, la quale presuppone, invece, la disponibilità del singolo ad anteporre alla propria utilità quella comune, talvolta richiedendo il sacrificio degli interessi personali in nome della collettività.

A tal riguardo, va osservato che, secondo Grozio, è al fine di fortificare la parentela che unisce gli uomini con un presidio più certo ${ }^{53}$ che essi si raggruppano in un unico luogo, dando vita ad una società più piccola rispetto all'umanità intera, vale a dire la società civile, che l'autore definisce anche Respublica ${ }^{54}$. In sostanza, per Grozio, la motivazione che induce alla nascita di tale società sarebbe anzitutto la tendenza di al-

\footnotetext{
52 In merito al rapporto tra legge naturale e legge civile, è interessante rilevare che cosa comandi, secondo il giovane Grozio, la Lex XIII, la quale afferma che quando le leggi appartenenti ad ordinamenti diversi non possono essere osservate simultaneamente, debba prevalere quella che ha maggiore dignità. Sul punto si rinvia ad H. GROTI, De jure praedae commentarius, cit., cap. II, p. 29. L'autore precisa, in proposito, che la dignità di una legge può essere stabilita in base all'origine e in base al fine. In base all'origine - e conformemente a quello che è l'ordine gerarchico delle fonti normative indicato dalle diverse $R e$ gulae - il diritto divino, e quindi anche il diritto naturale, primeggiano sul diritto romano, mentre il diritto umano (ossia lo jus gentium secundarium) prevale su quello civile. Secondo il fine, invece, ciò che riguarda il bene proprio è anteposto al bene altrui, il bene maggiore è anteposto al bene minore ed il male minore è anteposto al male maggiore. Si veda H. Groti, De jure praedae commentarius, cit., cap. II, p. 29.

${ }^{53}$ Cfr. H. GRotII, De jure praedae commentarius, cit., cap. II, p. 19.

${ }^{54}$ Cfr. H. GrotII, De jure praedae commentarius, cit., cap. II, pp. 19-20: "Haec igitur minor societas consensu quodam contracta boni communis gratia, i. e. ad se tuendam mutua ope et acquirenda pariter ea, quae ad vivendum necessaria sunt sufficiens multitudo, respublica dicitur et singuli in ea cives".
} 
cuni uomini a disattendere i comandi del diritto naturale. Si deve concludere, dunque, che la naturale socialità umana non conduca direttamente alla nascita dello stato politico, il quale sarebbe, piuttosto, frutto della capacità dell'uomo di stabilire patti e di rispettarli in vista di finalità prettamente utilitaristiche, vòlte a migliorare il tenore dell'esistenza. Non a caso, la visione antropologica groziana mostra come questi abbia ereditato dal calvinismo una concezione alquanto negativa della natura umana. Egli è convinto, infatti, che la ragione umana sia stata irrimediabilmente corrotta dal peccato originale, sicché sarebbe proprio a causa dell'ingenium corruptum di molti uomini che essi si mostrano incapaci di osservare il dettato delle norme di diritto naturale, non rispettando gli accordi che hanno contratto o attaccando le fortune e la vita altrui 55 . Stando alla ricostruzione proposta da Grozio, quindi, è per colpa della corruzione dell'uomo che fu necessario cercare una soluzione, affinché le leggi della società umana (humanae societatis leges) non venissero abbandonate ${ }^{56}$. Trova così conferma l'idea che lo Stato venga costituito appunto per garantire maggiormente l'obbedienza dei precetti naturali, non sufficientemente assicurati nella condizione prepolitica. È, dunque, lo scopo della tranquillità dell'ordine sociale e, quindi, della garanzia della salvezza individuale ad indurre i singoli ad entrare nella Respublica. Tanto più che per il bene duraturo del consorzio umano occorre non solo che gli esseri umani si attengano all'obbligo negativo di non ledere i propri simili, bensì è richiesto loro anche un impegno positivo a mettere in comune prodotti e servizi. Difatti, secondo il pensatore di Delft, gli uomini decidono di associarsi anche "ut multa, quae humanae vitae usum postular, distincta multorum opera commodius conferrentur" 57 . Ma lo scambio reciproco di beni è sempre più difficile nello stato di natura, poiché - nella ricostruzione di Grozio - con gli anni il numero degli esseri umani diventerebbe talmente grande che questi, vivendo separati in grandissimi spazi, non avrebbero più occasione di beneficiarsi a vicenda ${ }^{58}$.

${ }^{55}$ Cfr. H. GrotII, De jure praedae commentarius, cit., cap. II, p. 19: "His ita constitutis cum accideret ut multi, quae hominum quorundam ex corrupto ingenio malitia est, aut obligationibus non satisfacerent aut etiam fortunas alienas ipsamque vitam impeterent, idque plurimum impune ferrent, quia parati improvidos aut multi singulos adoriebantur, novo remedio opus fuit, ne humanae societatis leges invalidae destituerentur".

${ }^{56}$ Cfr. H. GROTII, De jure praedae commentarius, cit., cap. II, p. 19.

${ }^{57}$ Cfr. H. GrotII, De jure praedae commentarius, cit., cap. II, p. 19.

${ }^{58} \mathrm{Cfr}$. H. GrotII, De jure praedae commentarius, cit., cap. II, p. 19: "excrescente 
Ecco, dunque, quali sono le ragioni che, per Grozio, conducono alla nascita dello Stato attraverso il consenso di tutti in vista del bene comune, vale a dire "ad se tuendam mutua ope et acquirenda pariter ea, quae ad vivendum necessaria sunt sufficiens multitudo" 59 . L'obiettivo della consociazione politica, quindi, è quello di preservare se stessa e di procurarsi quanto sia necessario alla vita mediante l'opera mutua delle parti, ossia dei consociati. In tal modo, per l'autore, l'industria umana imita la natura, "quae universi conservationem foedere quodam rerum omnium firmavit"60. In altri termini, così come la conservazione dell'universo è garantita da una sorta di patto tra tutte le cose, che le induce all'amore verso i propri simili, egualmente gli uomini decidono di unirsi attraverso un accordo, per preservare al meglio la loro unione, con il beneplacito divino ${ }^{61}$. Tuttavia, Grozio non si sofferma ad analizzare le caratteristiche di tale accordo, limitandosi egli a ricordare come la società politica nasca non spontaneamente dalla naturale socialità degli uomini, bensì grazie alla loro capacità di sottoscrivere e rispettare dei contratti, "in vista di fini strettamente utilitaristici di migliore sopravvivenza: creare piccole comunità umane, i cui membri siano reciprocamente vincolati l'uno all'altro, e dove siano concentrati i mezzi di sussistenza"62. Da ciò si

praesertim in eam multitudinem hominum numero, ut longissimis discreti spatiis beneficiandi inter se occasiones amitterent".

${ }^{59} \mathrm{Cfr}$. H. GrotII, De jure praedae commentarius, cit., cap. II, p. 20.

${ }^{60}$ Cfr. H. Grotil, De jure praedae commentarius, cit., cap. II, p. 19. Qui Grozio sembra ricondurre l'armonia universale ad una 'sorta di foedus' tra tutti gli esseri del creato, in ragione del quale questi non perseguono solo il loro egoistico interesse, bensì tendono a procurare anche il bene ai propri simili. Il riferimento all'esistenza di una sorta di patto tra tutte le cose dell'universo, che le condurrebbe alla reciproca conservazione, compare già in un precedente luogo testuale del De jure praedae, quando il giovane autore sostiene che "at vero non satis conervationi operum suorum provisum Deus credidit, si suam duntaxat incolumitatem cuique commendaret, nisi et rem alteram alterius commodis vellet consulere, ut cuncta inter se velut aeterno foedere consentirent" (H. GROTII, De jure praedae commentarius, cit., cap. II, p. 11).

${ }^{61}$ Cfr. H. GrotII, De jure praedae commentarius, cit., cap. II, p. 20: "Initium huic ordini a Deo principe, qui omnem hunc mundum regit, cui nihil quod quidem in terris fiat esse acceptius philosophi prodiderunt, quam concilia coetusque hominum, quae civitates appellantur. Hanc legem, hoc jus, ut inquit Tullius, Juppiter ipse sanxit, ut omnia quae reipublicae salutaria essent legitima et justa haberentur. In hoc omnes fere gentes consentiunt, quae totum per orbem in tales conventus distributae conspiciuntur, ita ut vix hominum nomine digni videantur, qui ab isto instituto adhorrent, quare omnium probrorum quasi extremum est illud".

62 Cfr. P. Negro, Note a U. Grozio, I fondamenti del diritto. Antologia, cit., p. 263, n. 27 (corsivo nell'originale). 
può dedurre, quindi, che al momento dell'istituzione dello Stato, nel sottoscrivere il suo pactum fondationis i contraenti si impegnano in maniera mutua ad anteporre il benessere della collettività a quello individuale, in vista di una maggiore sicurezza per la propria esistenza ed i propri beni.

Ne consegue che la Respublica, avendo come fine la preservazione della società umana, costituisca il luogo di massima espressione della virtù sociale, che mira ad attribuire a ciascuno quello che gli spetta, sia nella sua forma di giustizia assegnatrice che di giustizia compensatrice ${ }^{63}$.

In particolare, mentre la giustizia assignatrix ascrive i possessi pubblici ai cittadini, che ne siano più degni, e distribuisce doveri e oneri in base alle forze di ognuno ${ }^{64}$, la giustizia compensatrix non solo ha cura di mantenere l'eguaglianza aritmetica (aequalitas) tra i singoli, ma remunera anche coloro che hanno ben meritato dalla patria onori e premi, punendo, invece, coloro che hanno nuociuto all'interesse comune $^{65}$. Quest'ultima forma di giustizia insegna appunto che nello Stato "in singulos fiunt ad cunctos pertineant" 66 , vale a dire che ciò che accade ai singoli riguarda tutta la comunità. Pertanto, la justitia compensatrix - sottolinea Grozio - da una parte, premia, ad esempio, un uomo che in battaglia abbia salvato un suo concittadino in virtù della legge di natura (Lex VI), per la quale i benefici vanno sempre ricompensati, dall'altra, non si limita a giudicare unicamente dei reati di lesa maestà, bensì chiede di perseguire anche quanti danneggino la collettività, come gli omicidi ed i falsari (Lex VII $)^{67}$.

Inoltre, proprio al fine di disciplinare la vita di tutti i cittadini all'interno dello Stato intervengono due nuove leggi di natura aventi come obiettivo specifico non la tutela del singolo o degli altri uomini, bensì del bene comune. La Lex VII, infatti, prescrive che "ut singuli cives caeteros tum universos, tum singulos non modo non laederent,

${ }^{63}$ Cfr. H. Grotil, De jure praedae commentarius, cit., cap. II, p. 20.

${ }^{64}$ Cfr. H. GrotII, De jure praedae commentarius, cit., cap. II, p. 20: "Nam assignatrix possessiones publicas pro dignitate cujusque dominis adscribit et officia onoraque in cives pro viribus dispertit".

${ }^{65}$ Cfr. H. GrotII, De jure praedae commentarius, cit., cap. II, p. 20: “compensatrix vero non tantum inter singulos aequalitatem tuetur, sed et beni de patria meritos honore et praemiis remuneratur et in commune nocentibus ad poenas utitur".

${ }^{66}$ Cfr. H. GRoTII, De jure praedae commentarius, cit., cap. II, p. 20.

${ }^{67}$ Cfr. H. GrotII, De jure praedae commentarius, cit., cap. II, pp. 20-21. 
verum etiam tuerentur" 68 , ossia comanda non solo il dovere passivo del neminem laedere verso i propri simili, assunti sia singolarmente che nella loro totalità, ma anche il dovere attivo di cura del prossimo. Se quest'ultima legge, quindi, ha ad oggetto la vita dei cittadini, la successiva, invece, ossia la Lex VIII, tutela i loro beni, sia posseduti privatamente che in comune, poiché stabilisce che "ut cives non modo alter alteri privatim aut in commune possessa non eriperent, verum etiam singuli tum quae singulis, tum quae universis necessaria conferrent" ${ }^{69}$. Non basta, dunque, che il cittadino non privi gli altri dei loro possessi, poiché gli è anche richiesto di contribuire attivamente a quanto sia necessario ai singoli e a tutta la collettività.

È evidente, quindi, che all'interno della società civile il rapporto tra i cives si definisca sulla base di doveri reciproci, che obbligano a non aggredire il corpo e i beni dell'altro, contribuendo, piuttosto, in maniera attiva al suo benessere. Ciò perché quando si diventa cittadini, sottoscrivendo il patto iniziale di fondazione dello Stato o manifestando una tacita adesione alla Respublica già costituita ${ }^{70}$, il proprio interesse individuale, difeso dalle prime due leggi di natura, confluisce nel benessere di tutti. In questo senso, si è soliti definire il bene di tutti come proprio bene ${ }^{71}$.

Inoltre, proprio il bene comune diviene il fine verso cui deve tendere l'attività di chi amministra lo Stato, vale a dire il magistrato. Più specificamente, la descrizione di tale figura segue, nell'ordine di esposizione del trattato, la formulazione delle Regulae $I V$ e $V$, le quali stabiliscono rispettivamente che "quidquid respublica se velle significaverit, id in cives universos jus est" 72 e che "quidquid respublica se velle significavit, id inter cives singulos jus est"73. Com'è chiaro, poiché i singoli decidono volontariamente di far parte della Respublica,

${ }^{68}$ Cfr. H. GRotII, De jure praedae commentarius, cit., cap. II, p. 21 (corsivo nell'originale).

${ }^{69}$ Cfr. H. GrotII, De jure praedae commentarius, cit., cap. II, p. 21 (corsivo nell'originale).

${ }^{70} \mathrm{Cfr}$. H. GrotiI, De jure praedae commentarius, cit., cap. II, p. 20.

${ }^{71}$ Difatti, per Grozio, "tamen ubi queritur de bono singulorum et de bono omnium, quorum utrumque recte bonum suum dicitur, si quidem et omnes unum sunt aliquid, bonum illud omnium potius esse debeat, quatenus ei scilicet et bonum inest singulorum, non aliter atque merces nisi nave servata salvae esse nequeunt" (H. GROTII, De jure praedae commentarius, cit., cap. II, p. 21).

72 Cfr. H. GrotII, De jure praedae commentarius, cit., cap. II, p. 23 (corsivo nell'originale).

${ }^{73}$ Cfr. H. GrotII, De jure praedae commentarius, cit., cap. II, p. 24 (corsivo nell'originale). 
ne consegue che per essi, sia singolarmente intesi (Regula IV), sia nella loro totalità (Regula $\mathrm{V})$, qualunque cosa lo Stato manifesti di volere valga come diritto.

Con il patto fondativo dello Stato, quindi, coloro che abbiano preso parte a tale accordo divengono membri della società politica, la quale ha, pertanto, sui singoli uno jus potestatis, "quae in legibus judiciisque conspicitur" 74 . In breve, i cittadini hanno il dovere di osservare le leggi e di sottoporsi al giudizio pubblico.

Infatti, secondo Grozio, le leggi civili non sono altro che la "voluntas universorum ad universos directa"75, ossia la volontà di tutti diretta a disciplinare il comportamento della totalità dei cittadini, sebbene egli sottolinei che tale volontà proceda da $\mathrm{Dio}^{76}$. Si potrebbe presumere, quindi, che la legge civile discenda da un atto di volontà del popolo sovrano, ancorché essa - precisa l'autore - ravvisi pur sempre la sua causa ultima nella volontà divina, per cui è stata giustamente denominata "inventum ac munus Dei" 77 . Il giudizio pubblico, invece, è la "voluntas universorum ad singulos directa boni publici intuitu"78, vale a dire la volontà di tutti, che dirige il singolo in vista dell'interesse comune. Lo Stato, infatti, interviene come mediatore nelle controversie tra cittadini, garantendo il rispetto dell' equità ed evitando che il singolo si attribuisca più del dovuto.

Alla luce di quanto sinora osservato, è chiaro che, secondo Grozio, il potere civile risieda originariamente nelle mani della stessa Respublica, ossia della totalità dei cittadini, "ut enim naturaliter singulis in se ac sua potestas est, ita in omnes resque omnium nisi omnium potestas esse non potest" 79 . Altrimenti detto, poiché il potere su di sé e sulle proprie cose appartiene al singolo, egualmente il potere su tutti e sulle cose di tutti non potrebbe esistere se non come potere di tutti. Ma non avendo ciascuno tempo da dedicare all'amministrazione degli affari civili, ed essendovi spesso situazioni che poche persone risolverebbero

${ }^{74}$ Cfr. H. Grotil, De jure praedae commentarius, cit., cap. II, p. 25.

${ }^{75}$ Cfr. H. Grotil, De jure praedae commentarius, cit., cap. II, p. 22. Questa sarebbe la definizione della lex proprie dicta.

${ }^{76}$ Cfr. H. GROTII, De jure praedae commentarius, cit., cap. II, p. 22.

77 Cfr. H. Grotil, De jure praedae commentarius, cit., cap. II, p. 22 (corsivo nell'originale).

${ }^{78}$ Cfr. H. Grotil, De jure praedae commentarius, cit., cap. II, p. 23.

${ }^{79}$ Cfr. H. GrotII, De jure praedae commentarius, cit., cap. II, p. 25. 
nel migliore dei modi, il popolo demanda tale potere, o una parte di esso, ad alcuni soggetti provenienti dal suo stesso corpo ${ }^{80}$.

Coloro che sono delegati alla gestione della cosa pubblica sono, appunto, i magistrati, i quali vengono istituiti in funzione non della propria utilità, bensì di quella dello Stato ${ }^{81}$. Tanto è vero che il contratto di mandato è regolato da due leggi di natura ${ }^{82}$, da cui si può dedurre una doppia obbligazione implicita nel rapporto di potere: " $\{$ Lex $X\} u t$ magistratus omnia gerat e bono reipublicae; altera: $\{$ Lex XI $\}$ ut quidquid magistratus gessit respublica ratum habeat"83. Se, quindi, da un lato il magistrato deve amministrare ogni cosa in base al bene della Respublica (Lex X), dall'altro la Respublica è tenuta a ratificare qualsiasi cosa il magistrato abbia deciso. In altri termini, i cittadini devono obbedienza a chi governa. Di conseguenza, le due successive Regulae, sancendo che il volere del magistratus costituisce diritto tanto per il singolo cittadino quanto per la collettività, presuppongono che chi eserciti il potere civile osservi sempre l'interesse collettivo. Tali regole prevedono, infatti, che " $\{$ Regula VI\} quod se magistratus velle significavit id in cives universos jus est; \{Regula VII $\}$ et, quod se magistratus velle significavit id in cives singulos jus est" 84 . Da ciò risulta che a mantenere la stabilità del corpo politico sia un equilibrio tra obblighi morali, sanciti dalle leggi di natura, poiché, mentre ai cittadini è comandata l'osservanza della volontà del magistrato, al magistrato è imposto, invece, di avere a cuore nell' amministrazione dello Stato il bene comune e non il suo interesse personale.

In conclusione, sulla base di quanto sinora osservato, si deve con-

${ }^{80} \mathrm{Cfr}$. H. GRotII, De jure praedae commentarius, cit., cap. II, p. 25: "Sed cursus sicut in re privata non ex nostris tantum actionibus obligamur aut commodum capimus, sed per illorum etiam operam, quos rebus nostris praefecimus, quia perinde est quod cuique facere licet id per se faciat an per alium, ita quia non cuivis de populo vacabat administrationi rerum civilium tempus impendere, quaedam etiam occurebant quae melius per paucos agerentur, evenit, quod et nunc in collegiis majoribus solet, ut societas pro jure potestatis, quam in singulos habet, nonnullis e suo numero istum latore partemve ejus aliquam mandaret".

${ }^{81}$ Cfr. H. GrotII, De jure praedae commentarius, cit., cap. II, pp. 25-26: "Nam magistratus, (...) at quatenus reipublicae sunt procuratores non ad suam, sed ad reipublicae utilitatem instituti sunt".

${ }^{82} \mathrm{Cfr}$. H. GrotII, De jure praedae commentarius, cit., cap. II, p. 26: "Quare leges due, quae contractui mandati naturaliter insunt, hic etiam reperuntiur".

${ }^{83}$ Cfr. H. GrotII, De jure praedae commentarius, cit., cap. II, p. 26 (corsivo nell'originale).

${ }^{84}$ Cfr. H. GrotII, De jure praedae commentarius, cit., cap. II, p. 26 (corsivo nell'originale). 
statare che, nel suo tentativo giovanile di sistemazione del diritto naturale, Grozio basi le relazioni intersoggettive sia nello stato di natura che nella società politica sulla fondamentale definizione della sfera del suum, ossia sull'eguale riconoscimento a tutti gli uomini di alcuni diritti fondamentali, tra cui il diritto alla vita ed alla proprietà. Sebbene in primis l'uomo si senta moralmente obbligato a difendere se stesso e ciò che gli appartiene, la miglior garanzia di una tranquilla fruizione dei suoi diritti gli è data dal rispetto del reciproco obbligo di non ingerenza nella sfera del suum altrui. Il dovere del neminem laedere si pone, quindi, in Grozio, quale presupposto di ogni consorzio umano. Ma proprio perché il comando naturale di astensione dai beni altrui non basta a garantire l'ordine sociale, in quanto esso è spesso disatteso da coloro che hanno una mente corrotta, si ha la necessità dell'ingresso nella Respublica. Una volta, però, che si scelga volontariamente di uscire dallo stato di natura per entrare nella società civile, il cittadino non sarà più soltanto vincolato a non nuocere al suo simile, ma dovrà anche partecipare attivamente al benessere della collettività. La ragione di ciò risiede in un significativo cambio di prospettiva del singolo, giacché con la partecipazione alla Respublica l'amor sui è sostituito da "una più complessa lungimiranza, in base alla quale può essere identificato un unitario bene collettivo, [che] viene giudicato la condizione stessa della propria personale utilità" 85 . In tale quadro generale, il giovane giusnaturalista costruisce il rapporto orizzontale tra cittadini, in cui rientrano le "mutuae actiones passionesque" 86 proprie della convivialità, sulla base di un dovere reciproco di non lesione e di condivisione, vale a dire di messa in comune di azioni e di beni.

Ed egualmente Grozio fa riposare su una reciproca obligatio anche il rapporto verticale tra governanti e governati ${ }^{87}$, poiché all'obbedienza

\footnotetext{
${ }^{87}$ A questo riguardo, Giulio Maria Chiodi rileva opportunamente come un rapporto politico, che sia fondato sulla correlazione tra dovere del cittadino e dovere delle istituzioni, prevenga la definizione della relazione tra governanti e governati nei termini di un privatistico do ut des, diversamente da quanto accadrebbe, invece, qualora si basassero i rapporti pubblici sulla relazione diritti-doveri. Sul punto si veda G.M. CHIODI, Precedenza dei doveri sui diritti umani, che peraltro sarebbe meglio definire diritti fondamentali, in G.M. CHIODI, "Europa. Universalità e pluralismo delle culture", Torino, Giappichelli, 2002, p. 152.
} ginale).

${ }^{85}$ Cfr. P. Negro, Note a U. Grozio, I fondamenti del diritto. Antologia, cit., p. 264, n. 28.

${ }^{86}$ Cfr. H. GRotII, De jure praedae commentarius, cit, cap. II, p. 14 (corsivo nell'ori- 


\section{comandata ai cittadini (Lex XI) si accompagna l'officium del magistrato di anteporre sempre l'interesse della Respublica al suo utile personale $(\text { Lex X) })^{88}$.}

\begin{abstract}
This paper moves from the examination of the different forms of law identified by Hugo Grotius in his De jure praedae (1604-08), in order to focus on the types of justice and the dynamics of duties, which operate within the Respublica here described. As is known, in this Grotian youthful work the supreme will of God represents the primary juridical source from which the peculiar classification of forms of law is outlined. This juridical voluntaristic structure acts as a precondition for the anthropological and political vision of the young Dutch thinker. Moreover, Grotius believes that justice is already manifested in the pre-political state as a social virtue involving the 'intermediate' position between someone's unfairly getting 'less' than is deserved and someone's unfairly getting 'more' at another's expense. Thus, the treatise
\end{abstract}

already contains a sketch of the Grotian doctrine of the suum, which will find further developments in De jure belli ac pacis. In particular, the problem of the limit of one's rights towards the others leads Grotius to face the reasons of the Respublica's birth. He assert that the Respublica, having as its purpose the preservation of human society, constitutes the place of the maximum expression of social virtue, which aims at attributing to each one what belongs to him, both in its form of justitia assignatrix and justitia compensatrix. Furthermore, with the entry into civil society the personal interest in common security prevails over everyone's selfish impulse. On such basis, both the relationship between citizens and the political relationship between the ruler and the ruled is conceived as founded on mutual duties.

${ }^{88}$ In proposito, si rinvia nuovamente a H. GROTII, De jure praedae commentarius, cit., cap. II, p. 25. La costruzione del rapporto politico governanti-governati e del rapporto civile fra $i$ cittadini in termini di reciproco dovere produrrà interessanti sviluppi nell'Illuminismo tedesco, attraverso la mediazione di alcuni dei principali rappresentanti del giusnaturalismo moderno di area germanica, quali Samuel Pufendorf e Christian Thomasius. Per un approfondimento della concezione del dovere nella dottrina filosofico-politica di questi due autori si rimanda rispettivamente a V. FIORILlo, Tra egoismo e socialità. Il giusnaturalismo di Samuel Pufendorf, cit., e a G. DiONI, Dalla stultitia alla sapientia. Il concetto di dovere nel giusnaturalismo di Christian Thomasius, con una prefazione di V. Fiorillo, Lecce, Pensa Multimedia Editore, 2009. Riguardo, invece, alla peculiare concezione dello Pflichtenstaat nella Germania illuministica del declinante Settecento si veda V. FIORILlo, Autolimitazione razionale e desiderio. Il dovere nei progetti di riorganizzazione politica dell'illuminismo tedesco, Torino, Giappichelli, 2000, passim. 